\title{
Homozygosity for the predominant Cys282Tyr mutation and absence of disease expression in hereditary haemochromatosis
}

\author{
D A Rhodes, R Raha-Chowdhury, T M Cox, J Trowsdale
}

Department of Human Immunogenetics, Imperial Cancer Research Fund, 44 Lincoln's Inn Fields, London WC2A 3PX, UK

D A Rhodes

J Trowsdale

Boyer Centre for Molecular Medicine, Department of Genetics, Yale University School of Medicine, 295

Congress Avenue, New Haven, CT 06536-0812, USA

R Raha-Chowdhury

Department of

Medicine, University

of Cambridge, Level 5, Addenbrooke's

Hospital, Hills Road, Cambridge CB2 2QQ, UK

D A Rhodes

T M Cox

Correspondence to

Dr Trowsdale.

Received 25 November 1996 Revised version accepted for publication 26 March 1997

\begin{abstract}
A candidate gene for hereditary haemochromatosis, HLA-H, has recently been presented. Two missense mutations in the HLA-H gene sequence are predicted to account for nearly $90 \%$ of all cases of the disease. The aim of this study was to correlate the presence of these missense mutations with the expressivity of the disease, as assessed by standard biochemical evaluation of serum iron parameters. Detection of the known mutations in haemochromatosis, Cys282Tyr and His63Asp, was undertaken in a large pedigree showing variable expression of the disease in successive generations. In three sibs with overt disease (one male, two female, aged 50 to 53 years), homozygosity for the predominant $G$ to $A$ transition (Cys282Tyr) in HLA-H was detected. However, homozygosity for this mutation was also detected in an asymptomatic male sib, aged 50, harbouring an identical genotype. The finding of an asymptomatic homozygous Cys282Tyr subject, haploidentical to affected sibs, indicates that clinical expression of symptomatic disease is variable, even in middle aged Cys282Tyr homozygotes. This has profound implications for the future use of genetic screening for haemochromatosis. ( Med Genet 1997;34:761-764)
\end{abstract}

Keywords: hereditary haemochromatosis; HLA; iron metabolism

Hereditary haemochromatosis is a prevalent, inborn error of iron metabolism. Homozygosity for the predisposing gene impairs regulation of iron balance by the intestine, leading to widespread deposition of iron and tissue injury. This may manifest as arthritis, liver disease, diabetes mellitus, and cardiomyopathy. Cirrhosis of the liver is common and the disease is associated with an increased risk of hepatocellular carcinoma, which accounts for about $30 \%$ of the deaths in affected homozygotes. ${ }^{1}$ Typically, haemochromatosis is associated with non-specific symptoms including weakness, malaise, and decreased libido in middle life. This lack of specificity probably results in underdiagnosis of this disease, which is predicted to affect 1 in 300 people of northern European origin. $^{23}$

Recently, a convincing candidate gene for haemochromatosis has been presented. ${ }^{4}$ The cloned gene lies within a region of extensive linkage disequilibrium extending $5 \mathrm{Mb}$ telomerically from the major histocompatibility gene complex on the short arm of chromosome 6. It represents a new HLA class 1 related gene and has been named HLA-H. Analysis of the gene sequence from patients identified two missense mutations which are predicted to affect significantly the tertiary structure and therefore the function of the cognate protein. A single, homozygous $\mathrm{G}$ to $\mathrm{A}$ transition, causing a cysteine to tyrosine amino acid change at position 282 of the protein, was detected in $83 \%$ of haemochromatosis patients. This observation fits well with the previous genetic studies, which predicted that a single mutation would predominate in this disease. ${ }^{56} \mathrm{~A}$ second $\mathrm{C}$ to $\mathrm{G}$ mutation, resulting in a change from histidine to aspartic acid at position 63, accounted for a further $4 \%$ of haemochromatosis patients. ${ }^{4}$

We have investigated an extensive pedigree affected by haemochromatosis using HLA typing, microsatellite polymorphisms, by biochemical testing of serum iron parameters, and by liver biopsy (table 1). The diagnosis of haemochromatosis was based on these investigations and the results are shown, together with the pedigree structure, in fig 1 . Of particular interest were the results of the biochemical evaluation of subject II.6, which were apparently anomalous with the results of the genetic analysis. He showed no signs of iron overload, with a wide discrepancy between his transferrin saturation, which on two occasions touched the upper limits of the normal range, and his serum ferritin, the results of which remained very low on all three occasions. He nevertheless carried an identical genotype in the HLA region to his symptomatic sibs, all of whom had overt disease diagnosed at a similar age. Since no recombined chromosomes were detected, we concluded that he was a non-expressing homozygote. $\mathrm{He}$ is accordingly represented by a grey symbol in fig 1, indicating that he is phenotypically negative, yet homozygous for the predisposing disease allele. In addition, the transferrin saturation level of his son, III.3, aged 21 years, was just above the normal range at $61 \%$, together with serum iron and ferritin levels within the normal range. This may reflect partial heterozygous expression.

We analysed members of this family for the presence of the recently described missense mutations in the HLA-H gene, predicted to account for $87 \%$ of all cases of haemochromatosis. ${ }^{4}$ From the haplotype 
Table 1 Results of the biochemical investigation of serum iron parameters in a three generation family with hereditary haemochromatosis

\begin{tabular}{|c|c|c|c|c|c|}
\hline Subject & Agef & $\begin{array}{l}\text { Ferritin } \\
(\mu g / l)\end{array}$ & $\begin{array}{l}\text { Transferrin } \\
\text { saturation }\end{array}$ & $\begin{array}{l}\text { Serun iron } \\
(\mu m o l l l)\end{array}$ & Liver \\
\hline I. 1 & 63 & & $97 \%$ & & + \\
\hline I. 2 & 80 & 225 & $27 \%$ & & \\
\hline II. 1 & 62 & 192 & $70 \%$ & & \\
\hline II. 2 & 53 & 670 & $40 \%$ & & + \\
\hline II. $3^{\star}$ & 52 & 4700 & $77 \%$ & 40.5 & + \\
\hline II. 4 & 50 & 640 & $60 \%$ & 42 & + \\
\hline II. 5 & 48 & $157 / 258$ & $76 / 57 \%$ & & \\
\hline $\begin{array}{l}\text { II. } 6 \dagger \\
\text { II. } 7\end{array}$ & $42 / 44 / 50$ & $23 / 28 / 28$ & \multicolumn{2}{|c|}{ II.7 } & \\
\hline II. 8 & 45 & 7 & $12 \%$ & 10.5 & \\
\hline \multicolumn{6}{|l|}{ III. 1} \\
\hline III. 2 & & 79 & & & \\
\hline III.3 & 21 & 138 & $61 \%$ & 29.2 & \\
\hline \multicolumn{6}{|c|}{ Normal ranges $\delta$} \\
\hline Male & & $15-235$ & $16-60 \%$ & $14-32$ & \\
\hline Female & & $12-150$ & $16-50 \%$ & $10-28$ & \\
\hline
\end{tabular}

*Proband results at diagnosis. †Iron values of asymptomatic subject II. 6 remained within the nor mal range over a seven year period (1988-1995). $¥$ Age at time of testing. §Edwards and Kushner. ${ }^{10}$

analysis, it was predicted that all second generation subjects with overt disease would be homozygous for the predominant Cys282Tyr mutation ( $G$ to A transition). The result of single nucleotide primer extension analysis (fig $2 \mathrm{~A})^{7}$ confirmed this prediction and showed that the standard biochemical procedures had accurately discriminated the disease state in most cases shown in fig 1 . This was not the case for the asymptomatic subject II.6, who was also homozygous for the disease allele. $\mathrm{He}$ is indeed a non-expressing homozygote who must be considered to be at risk for the development of overt haemochromatosis.

Before testing for the disease related alleles of HLA-H, the raised serum transferrin saturation level of III.3, the son of the non-expressing homozygote II.6, could not be readily explained. We screened the family for the His63Asp mutation and this result (fig 2B) showed that both II.8, the wife of II.6, and III.3, their son, were heterozygotes for this allele. III. 3 was thus a compound heterozygote for haemochromatosis and carries both missense mutations so far associated with the disease. This genotyping at the HLA-H locus accounted for his raised serum transferrin saturation level and showed that this subject, in the third generation, is also at risk for the development of overt haemochromatosis.

The serum iron parameters of the asymptomatic subject II. 6 remained consistently normal over a seven year period to the age of 50 . His brother, II.3, was referred at the age of 49 with malaise and abnormal serum liver related enzymes and liver biopsy showed cirrhosis with grade 4 iron deposits indicating haemochromatosis. $^{8} \quad$ Subsequent investigations showed that two sisters, aged 53 and 50 , both of whom had borne children, had abnormal serum parameters of iron metabolism and liver biopsy confirmed grade 3 siderosis. They each required $>25$ venesections to remove excess iron stores.

In the pedigree reported, haplotype analysis had alerted us to the possibility that II. 6 was a non-expressing homozygote who was at risk of haemochromatosis in the future. Our findings are consistent with the hypothesis of Feder et $a l^{4}$ that the Cys282Tyr mutation in HLA-H is the causative mutation responsible for most cases of haemochromatosis, but they also indicate that expression of the disease is strikingly variable. This variability in expression might be related to age, ${ }^{9} \operatorname{sex}^{210}$ or nutritional factors such as meat intake or alcohol consumption. ${ }^{11}$ Women are regarded as being less prone to iron overload because of iron loss during menstruation and childbirth ${ }^{210}$; however, the two multiparous sisters of II. 6 both developed overt disease. Although classical haemochromatosis shows marked sex limited expression of the disease, variation induced by gender cannot account for differences in expressivity in this pedigree. II. 6 is not known to have any dietary idiosyncrasies and had not been a regular blood donor. It is interesting to note that of the two members of the family reported previously who were unavailable for further study, the HLA identical older sister (II.5) was iron deficient and had coeliac disease, a malabsorption syndrome associated with sensitivity to gluten. The presence of these symptoms was investi-

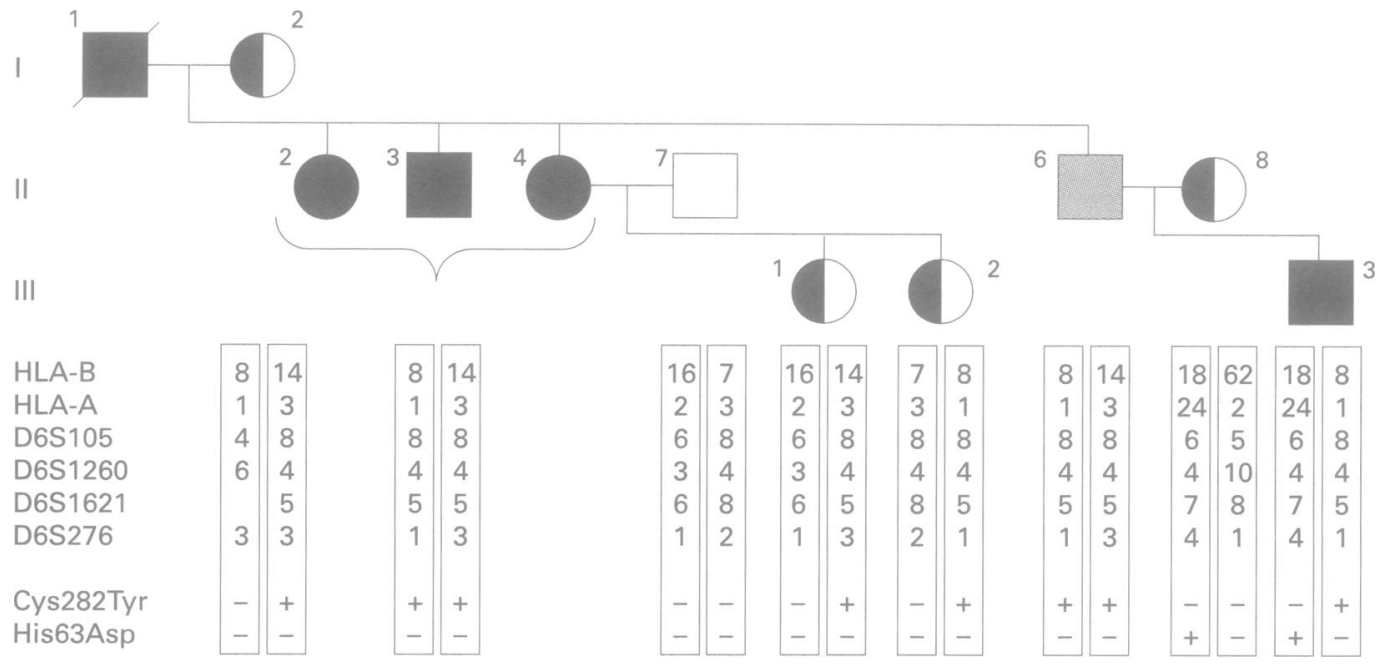

Figure 1 Pedigree structure and summary of genetic analysis using HLA class I serological markers and polymorphic microsatellites. Dark symbols signify a diagnosis of hereditary haemochromatosis based on the results shown in table 1 and haplotype analysis, except in the case of II. 6 who is represented in grey, as discussed in the text. Genotyping results at the $H L A-H$ gene locus are presented below the haplotypes. + indicates the presence of the mutant allele and - the presence of the wild type allele. The HLA-H gene is located between D6S1260 and D6S276. 

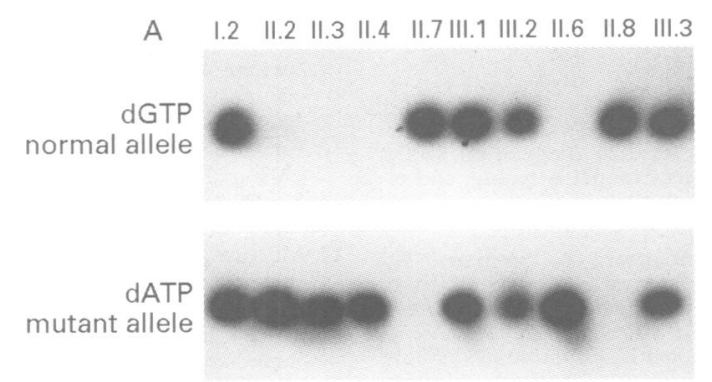

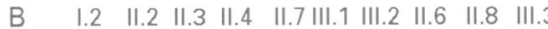

normal allele

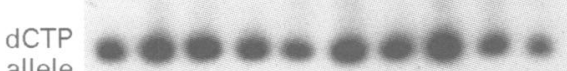

dGTP

mutant allele

Figure 2 Single nucleotide primer extension (SNuPE) analysis to detect nucleic acid base substitutions.' $(A)$ Detection of the $G$ to $A$ transition, resulting in the Cys282Tyr amino acid change in the $H L A-H$ protein. Incorporation of labelled dGTP indicates the presence of the normal allele, while incorporation of dATP indicates the mutant allele. Subject I. 2 is shown to be heterozygous while all second generation subjects with diagnosed

haemochromatosis have the homozygous mutant allele. The asymptomatic subject II. 6 also carries this homozygous base change. (B) SNuPE analysis for the His63Asp amino acid change. Incorporation of labelled $d C T P$ denotes the normal allele and incorporation of dGTP denotes the mutant allele. Subjects II. 8 and III. 3 are heterozygous at this locus, while all other members of the pedigree are homozygous for the normal allele.

gated in II.6, who had no history of anaemia and showed no abnormalities in liver function tests or evidence of gastrointestinal disturbance. Furthermore, IgA and IgG antigliadin antibodies were not raised and the coeliac specific antiendomyseal IgA, while on an unrestricted diet, was only weakly positive. We concluded that coeliac disease was absent in II.6 and the variability in the clinical expression of haemochromatosis could not be accounted for by the presence of this or any other disease. It may be of considerable importance to determine the reasons for this variability in penetrance.

The raised serum transferrin saturation level of III.3, the son of II.6, was unexplained. Although this level is only slightly above the normal range, we initially considered him to be heterozygous with partial expression of haemochromatosis. ${ }^{12}$ It is noteworthy that III. 3 is a compound heterozygote with abnormal serum transferrin saturation at the age of 21 years. II.8, the mother of III.3, carries the His63Asp variant (associated with an HLAA24, HLA-B18 class I haplotype) yet her serum iron parameters are in the anaemic range (table 1). These data are consistent with the His63Asp variant having pathological effects on iron metabolism when coinherited with the Cys282Tyr allele and may indicate that compound heterozygotes for the two prevalent mutations in HLA-H are more susceptible to iron overload than homozygotes.

It is noted that Feder $\mathrm{et} \mathrm{al}^{4}$ observed a relative risk of 0.0057 for compound heterozygotes versus Cys282Tyr homozygotes, based on their population data. Beutler et $a l^{13}$ also recorded a reduced penetrance for compound heterozygotes and similarly suggested that the His63Asp variant may only be a deleterious mutation when inherited in trans with the Cys282Tyr allele.

The question of variability in disease expression of haemochromatosis by evaluation of serum iron parameters has been addressed previously. Family studies and HLA typing have been used to assign non-expression to putative homozygotes and partial expression to putative heterozygotes. ${ }^{12}{ }^{14} 15$ Evidence for genetic heterogeneity in the genetic factors underlying disease expression has also been discussed. ${ }^{16}$ Added to this confusing picture is the detection of $\operatorname{sex}^{210}$ and age $e^{9}$ related variability in disease expression and the possibility of genetic recombination accounting for discordant results in HLA identical sibs. ${ }^{14}$ In addition, both concordant expression of iron storage between sibs ${ }^{17}$ and intrafamilial variation in expression of haemochromatosis ${ }^{18}$ have been presented.

In this report, we have correlated the presence of the specific missense mutations described by Feder et $a l^{4}$ with clinical expression of iron overload in a single three generation family. We have shown for the first time that in the absence of genetic recombination and of any known environmental factors which may influence iron metabolism, clinical expression of the symptoms of iron overload are extremely variable. The results are consistent with intrafamilial variation in disease expression and are not consistent with the hypotheses regarding sex and age limitations in disease expression. These results may also shed some light on the role of the His63Asp variant in haemochromatosis.

Despite the formidable genetic analysis presented by Feder et $a l,{ }^{4}$ it remains a formal possibility that the haemochromatosis gene lies elsewhere within the extensive region of linkage disequilibrium. ${ }^{19}$ This extensive linkage disequilibrium could also underlie a complex relationship of the region with the disease, involving loci in addition to HLA-H. ${ }^{20}$ There has been extensive duplication of class I-like sequences in the telomeric part of the MHC and functional degeneracy of these related genes may be manifest as coinheritance with particular haplotypes. In order to account for the genetic heterogeneity detected in their analysis, Feder et $a l^{4}$ suggested that another locus, not necessarily MHC linked, is involved in the disease.

Although the results presented here are fully consistent with the candidacy of HLA-H, they document that homozygosity for the predisposing allele, Cys282Tyr, may occur in the complete absence of clinical disease, even within members of an extensive pedigree with multiple affected subjects. The identification of a homozygous male, now aged 50, with no sign 
of iron overload, challenges the utility of population or presymptomatic screening by genetic testing for haemochromatosis.

This work was supported in part by the Wellcome Trust. We are grateful to all the family members for their cooperation, to $\mathrm{Dr}$ Mark Worwood and to Dr Val Joycey for HLA typing, and to Joan Grantham for expert secretarial help.

1 Niederau C, Fischer R, Sonnenberg A, Stremmel W, Trampish HJ, Strohmeyer G. Survival and causes of death in cirrhotic and in non-cirrhotic patients with primary haemochromatosis. $N$ Engl f Med 1985;313:1256-62.

2 Edwards CQ, Griffen LM, Goldgar D, Drummond C, Skolnick MH, Kushner JP. Prevalence of hemochromatosis among 11,065 presumably healthy blood donors. $N$ Engl F Med 1988;318:1355-62.

3 Leggett BA, Halliday JW, Brown NN, Bryant S, Powell LW. Prevalence of haemochromatosis amongst asymptomatic Australians. Br $\mathcal{F}$ Haematol 1990;74:525-30.

4 Feder JN, Gnirke A, Thomas W, et al. A novel MHC class I-like gene is mutated in patients with hereditary I-like gene is mutated in patients with

5 Jazwinska EC, Pyper WR, Burt MJ, et al. Haplotype analysis in Australian hemochromatosis patients: evidence for a in Australian hemochromatosis patients: evidence for a predominant ancestral haplotype exclusively associated
with hemochromatosis. Am f Hum Genet 1995;56:428-33. with hemochromatosis. Am f Hum Genet 1995;56:428-33.
Raha-Chowdbury R, Bowen DJ, Stone C, et al. New Raha-Chowdbury R, Bowen DJ, Stone C, et al. New
polymorphic microsatellite markers place the haemochromatosis gene telomeric to D6S105. Hum Mol Genet 1995; 4:1869-74.

7 Kuppuswamy MN, Hoffmann JW, Kasper CK, Spitzer SG, Groce SL, Bajaj SP. Single nucleotide primer extension to detect genetic diseases: experimental application to hemophilia B (factor IX) and cystic fibrosis. Proc Natl Acad Sci USA 1991;88:1143-7.

8 Dokal I, Lord D, Rhodes D, Bydder G, Cox T. Detection of hereditary haemochromatosis in an HLA-identical pedi- gree showing discordance between HLA class I genes and the disease locus. Hum Genet 1991;88:209-14.

9 Bassett ML, Halliday JW, Ferris RA, Powell LW. Diagnosis of hemochromatosis in young subjects: predictive accuracy of biochemical screening tests. Gastroenterology 1984;87: 628-33.

10 Edwards CQ, Kushner JP. Screening for haemochromatosis N Engl f Med 1993;328:1616-20.

11 Lynch SR, Skikne BS, Cook JD. Food iron absorption in idiopathic hemochromatosis. Blood 1989;74:2187-93.

12 McLaren CE, Gordeuk VR, Looker AC, et al. Prevalence of heterozygotes for hemochromatosis in the white population of the United States. Blood 1995;86:2021-7.

13 Beutler E, Gelbert T, West C, et al. Mutation analysis in hereditary haemochromatosis. Blood Cells, Molecules and Diseases 1996;22:187-94.

14 Powell LW, Summers KM, Board PG, Axelsen E, Webb S, Halliday JW. Expression of hemochromatosis in homozygous subjects: implications for early diagnosis and mozygous subjects: implications for early diagn

15 Dadone MM, Kushner JP, Edwards CQ, Bishop DT, Skolnick MH. Hereditary hemochromatosis. Analysis of laboratory expression of the disease by genotype in 18 pedigrees. Am F Clin Pathol 1982;78:196-207.

16 Muir WA, McLaren GD, Braun W, Askari A. Evidence for heterogeneity in hereditary hemochromatosis: evaluation of 174 persons in nine families. Am $\mathcal{F}$ Med 1984;76:806-14

17 Crawford DH, Halliday JW, Summers KM, Bourke M Powell LW. Concordance of iron storage in siblings with genetic haemochromatosis: evidence for a predominantly genetic effect on iron storage. Hepatology 1993:17:833-7.

18 Adams PC. Intrafamilial variation in hereditary haemochromatosis. Dig Dis Sci 1992;37:361-3.

19 Little P. Woman's meat, man's poison. Nature 1996;382. 494-5.

20 Jazwinska EC, Cullen LM, Bustfield F, et al. Haemochromatosis and HLA-H. Nat Genet 1996;14:249-52. 